\title{
Dense and Nearly Jammed Random Packings of Freely Jointed Chains of Tangent Hard Spheres
}

\author{
Nikos Ch. Karayiannis and Manuel Laso* \\ Institute for Optoelectronics and Microsystems (ISOM) and ETSII, UPM José Gutiérrez Abascal, 2 E-28006 Madrid, Spain
}

(Received 26 July 2007; revised manuscript received 2 October 2007; published 7 February 2008)

\begin{abstract}
Dense packings of freely jointed chains of tangent hard spheres are produced by a novel Monte Carlo method. Within statistical uncertainty, chains reach a maximally random jammed (MRJ) state at the same volume fraction as packings of single hard spheres. A structural analysis shows that as the MRJ state is approached (i) the radial distribution function for chains remains distinct from but approaches that of single hard sphere packings quite closely, (ii) chains undergo progressive collapse, and (iii) a small but increasing fraction of sites possess highly ordered first coordination shells.
\end{abstract}

DOI: 10.1103/PhysRevLett.100.050602

PACS numbers: 05.20.- $-\mathrm{y}, 61.20 .-\mathrm{p}$

Maximally random jammed (MRJ) packings of identical spheres has been the subject of extensive analytical and numerical work, and a considerable body of knowledge has been collected over the last two decades (see [1-3] and references therein). It seems, however, that work has been concentrated on packings of single spheres while dense packings of chains have received comparatively little attention [4-6]. In this Letter we present numerical results about the packing of freely jointed chains of tangent hard spheres at volume fractions very close to the MRJ state [2] in three-dimensional Euclidean space. The systems investigated consisted of ensembles of freely jointed chains of tangent hard spheres of unit diameter and lengths $N=12$ or $N=24$ with a total of 1200 interaction sites. Both $N=$ 12 and $N=24$ lie deep in the infinite-chain asymptotic regime regarding packing and local structure, although chain dimensions have not reached asymptotic behavior. Persistence length, computed as $\left\langle R^{2}\right\rangle / 2 N+0.5$ [7] at $\varphi=$ 0.10 is 1.59 for $N=12$ and 1.80 for $N=24$.

Several algorithms for packing single spheres exist, some of them able to generate random packings up to the MRJ density [8] $\varphi_{\mathrm{ss}} \approx 0.64$ (subindices ss refer to single spheres, hsc to hard sphere chains). Although computationally demanding, recent algorithms can produce very dense packings with such efficiency that extremely large packings of up to a million spheres, necessary to investigate some subtle structural features, are within reach [9]. It is somewhat surprising that packings of hard-sphere chains have received little attention, although they represent a valuable departing point for perturbation work [10], in addition to having richer structure than single spheres. The main hurdle is the substantial computational difficulty associated with the generation and relaxation of packings of hard spheres which are close to the MRJ state and simultaneously satisfy the holonomic constraints defining chain connectivity. Molecular dynamics (MD) methods are notoriously inefficient at compacting ensembles of linear chains because of increasingly sluggish dynamics and large relaxation times as chain length increases. Monte Carlo (MC) schemes have been shown [11] to be a preferable alternative. Preliminary tests of available algorithms for generating dense, off-lattice chain packings clearly showed their inadequacy close to the MRJ state. No single algorithm working in isolation was able to generate random chain packings with anywhere near acceptable efficiency at $\varphi_{\text {hsc }}>0.58$ (though some recent generation algorithms are very efficient at intermediate to high volume fractions [12]). However, by using a carefully chosen combination and modification of several state-of-the-art MC algorithms for efficient structural relaxation of both large and small structural features we succeeded in producing very dense packings up to the neighborhood of the MRJ state. Specifically, the proposed MC scheme consists of the following set of moves: intermolecular end bridging $(1 \%)$, reptation (33\%), intramolecular end bridging $(1 \%)$, continuum configurational bias (30\%), flip (25\%), and end rotation (10\%) [13]. All moves were modified to handle dense systems of athermal chains while guaranteeing strict microscopic reversibility and preservation of configuration space volume for the underlying Markov chain [14]. Chain-connectivity altering moves and configurationbiased moves achieve long-range relaxation. The remaining, local moves relax structure at short range and act as a "lubricant" which greatly enhances overall efficiency [13]. Extreme care was exerted in minimizing the dependence on protocol: chain packings at increasingly higher densities were generated by entirely different protocols (cell compression, akin to standard isobaric MC moves, intercalated in long stretches of isochoric moves; relaxation of configurations of chains of length $N$ obtained by splitting prerelaxed configurations of chains of length $2 N$ ). All MC simulations were performed in cubic simulation cells with periodic boundary conditions applied in all dimensions. Typical lengths for relaxation runs were of $O\left(10^{11}\right)$ MC steps, each run taking $O\left(10^{7}\right)$ CPU seconds on modern processors. The results presented below are based on ensembles of configurations obtained from fully relaxed trajectories and in which protocol dependence could not be detected by standard measures [13], nor by the sensitive crystallographic norms to be defined below. The packing 


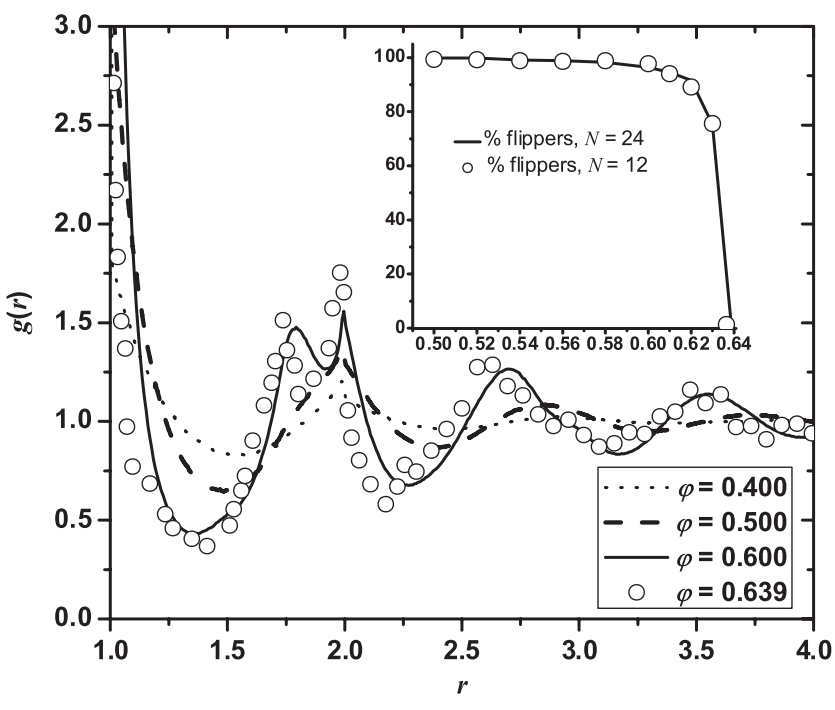

FIG. 1. Radial pair distribution function $g_{\text {hsc }}(r)$ for chains at several densities. $g(r)$ is calculated by taking into account all intra- and intermolecular neighbors (including the bonded ones) around the reference hard sphere. Inset: percentage of flipper spheres as a function of packing density.

density at the MRJ state (obtained by extrapolation analogous to that in [2]) for freely jointed chains of tangent hard spheres was found to be $\varphi_{\mathrm{hsc}}=0.638 \pm 0.004$ for both $N=12$ and 24, in agreement with the value for single hard spheres. It seems to be possible to pack freely jointed chains of hard spheres just as densely as, but not more densely than, individual spheres, even for quite long chains [14]. The evolution of the total radial pair distribution function, $g_{\mathrm{hsc}}(r)$, as density is increased is shown in Fig. 1 [15]. Unlike in hard sphere packings, rattlers [8] cannot exist in chain packings. For chains, the analog of a rattler is a sphere that can perform a differential "flip"-like movement (clockwise, counterclockwise or both) without incurring overlaps. The inset in Fig. 1 shows how the fraction of such "flipper" spheres precipitously declines as the MRJ state is approached. At the same time, $g_{\text {hsc }}(r)$ increasingly resembles $g_{\mathrm{ss}}(r)$ (Fig. 2), although the double tangency constraint has a small but noticeable effect on $g(r)$, especially at $r \approx 1$. Bond (Fig. 3) and torsion angle (Fig. 4) distributions undergo major changes as packing density increases. Characteristic bond and torsion angles are favored at high density. As a consequence, chain size measures (end-to-end distance, $\left\langle R^{2}\right\rangle$, and radius of gyration $\left.\left\langle R_{g}^{2}\right\rangle\right)$ undergo a continuous decrease (Fig. 5); i.e., more compact chain conformations are preferred at high volume fractions. Specifically, at $\varphi=0.1\left\langle R^{2}\right\rangle=26.2 ;\left\langle R_{g}^{2}\right\rangle=$ 4.00 for $N=12$, and $\left\langle R^{2}\right\rangle=62.4 ;\left\langle R_{g}^{2}\right\rangle=9.66$ for $N=$ 24 ; and at $\varphi=\varphi_{\mathrm{hsc}}=0.638,\left\langle R^{2}\right\rangle=15.9 ;\left\langle R_{g}^{2}\right\rangle=2.68$ for $N=12$, and $\left\langle R^{2}\right\rangle=34.6 ;\left\langle R_{g}^{2}\right\rangle=5.78$ for $N=24$. Kratky representations $F(k) k^{2}$ vs $k$ of the intramolecular form factor $F(k)$ versus wave number $k$ for $N=24$ are shown in the inset of Fig. 5. For the intermediate $k$ regime

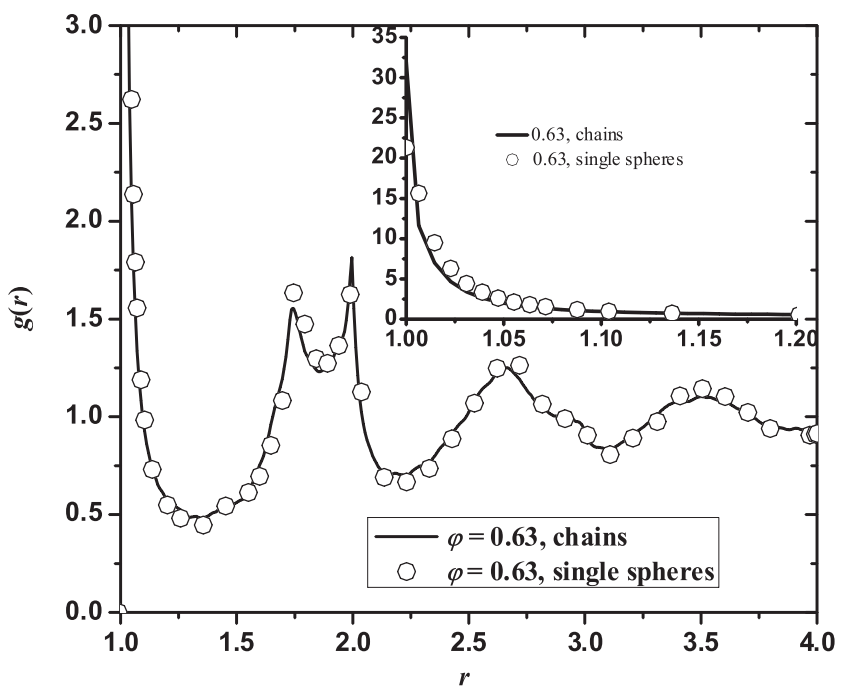

FIG. 2. Comparison between $g_{\mathrm{hsc}}(r)$ and $g_{\mathrm{SS}}(r)$ at $\varphi=0.63$. Inset: $g_{\mathrm{hsc}}(r)$ and $g_{\mathrm{ss}}(r)$ near contact.

at the lower packing density $\varphi=0.30$ chains adopt an isolated self-avoiding pattern as shown by a monotonic increment of $F(k) k^{2}$ with $k$. In contrast, at $\varphi=0.63$ the presence of a plateau in $F(k) k^{2}$ is apparent as expected for meltlike chains at large $N$. For an ideal chain the intermediate- $k$ scaling should follow $F(k) k^{2} \cong 12 N /\left\langle R^{2}\right\rangle$ [16]; consequently for Gaussian chains $\left(\left\langle R^{2}\right\rangle=N l^{2}\right.$, where $l$ is the bond length) a plateau value of 12 is predicted, quite higher than the simulation value of around 6.8, suggesting that the simulated chains depart from ideal ones due to intramolecular excluded volume interactions. If we consider an "effective length", extracted from simulation data of $\left\langle R^{2}\right\rangle$ (see Fig. 5), then the plateau value of the ideal chains drops to approximately 8.1 , about $15 \%$ higher than

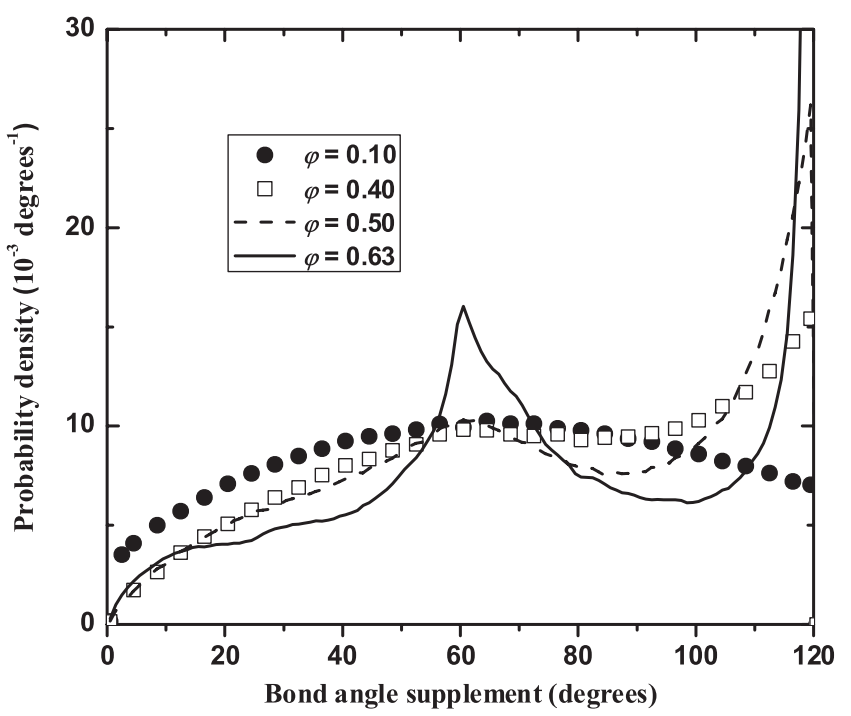

FIG. 3. Supplement bond angle distributions at increasing packing densities. 


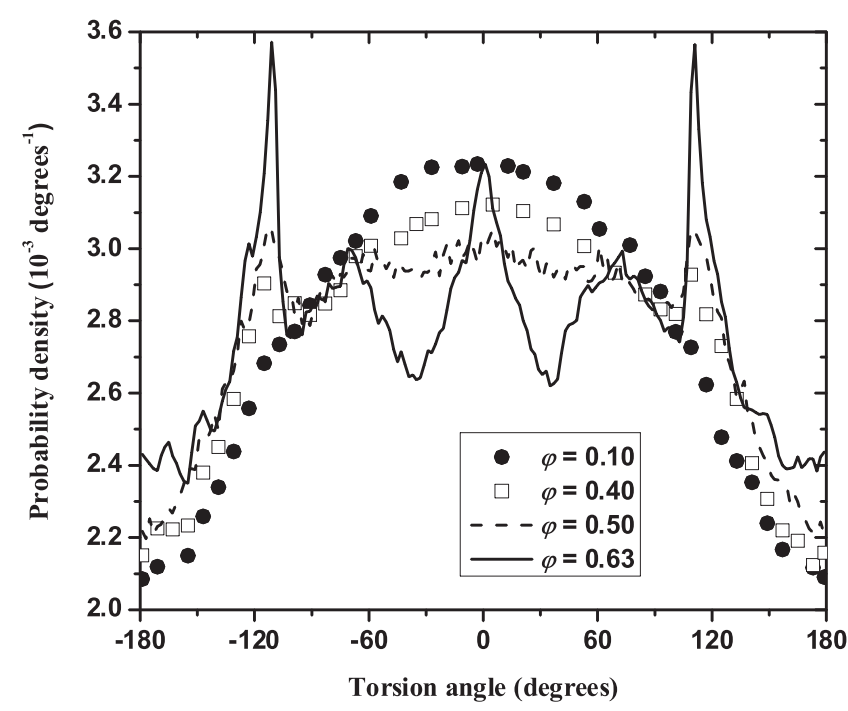

FIG. 4. Torsional angle distributions at increasing packing densities.

the Kratky value extracted from our simulations. The above finding, suggesting deviations from the ideality hypothesis, is in qualitative agreement with recent work by Semenov and co-workers [17].

A number of measures have been introduced in the literature $[1,18]$ to characterize local structure. While $g(r)$ contains the full radial information about the distribution of pairs of sites, rotational invariant combinations of spherical harmonics have been used to characterize the orientational part. Such measures can be used to address questions related to the incipient appearance of order as the MRJ state is approached [19].

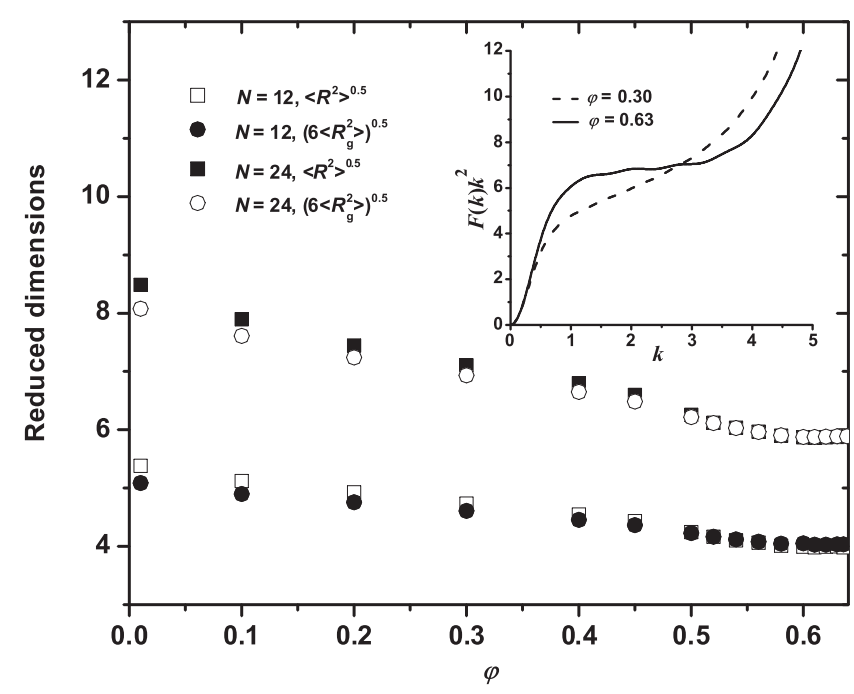

FIG. 5. Radius of gyration and end-to-end distance as a function of packing density. Error bars are smaller than symbol size. Inset: Kratky plot $F(k) k^{2}$ vs $k$ of the intramolecular form factor, $F(k)$, versus wave number, $k$, for $N=24$ at $\varphi=0.30$ and 0.63 .
We introduce a type of measures (norms) inspired by the basic principle of the characteristic crystallographic element (cce) [20]. These norms univocally quantify the degree to which the environment of a given sphere is more fcc-like or more hcp-like, both radially and orientationally. Given that the characteristic crystallographic element (fingerprint) of an hcp environment is a single $\overline{6}$ inversion axis, we define the hcp-cce norm $\varepsilon_{i}^{\text {hcp }}$ for the $i$ th site in a given configuration as

$$
\varepsilon_{i}^{\mathrm{hcp}}=\min _{\text {orientation of } \overline{6} \text { axis }}\left[\frac{1}{2 \sqrt{15}} \sqrt{\sum_{j=1}^{5} \sum_{k=1}^{12}\left(\mathbf{r}_{k}-\mathbf{r}_{k}^{\mathrm{hcp}}\right)^{2}}\right],
$$

where $\mathbf{r}_{k}$ is the position vector of the $k$ th first neighbor around the $i$ th site (obtained by Voronoi tesselating each configuration), $j$ is a counter over the elements of the $\overline{6}$ group and $\mathbf{r}_{k}^{\text {hcp }}$ is the position vector of the $k$ th site out of the 12 sites of the disheptahedral coordination polyhedron if the environment of the given ith site were perfect hcp. The key point in the definition (1) is that although $\mathbf{r}_{k}^{\text {hcp }}$ is of course not known for a given site, when constructing (1), the orientation of the $\overline{6}$, which defines $\mathbf{r}_{k}^{\text {hcp }}$, is to be found by a search in the two-parameter space of azimuthal and polar angles which define the $\overline{6}$ axis orientation: for each site in each MC frame a local $\overline{6}$ axis is determined that minimizes the double sum. $\varepsilon_{i}^{\text {hcp }}$ is set to this minimum. The norm distribution $\xi\left(\varepsilon^{\mathrm{hcp}}\right)$ is then obtained from the set of $\varepsilon_{i}^{\text {hcp }}$ for all sites, cumulated over the ensemble and properly normalized. Analogously, the fcc-cce norm for site $i, \varepsilon_{i}^{\mathrm{fcc}}$, is:

$$
\varepsilon_{i}^{\mathrm{fcc}}=\min _{\text {orientation of } \overline{3} \text { axes }}\left[\frac{1}{4 \sqrt{15}} \sqrt{\sum_{m}^{4} \sum_{j=1}^{5} \sum_{k=1}^{12}\left(\mathbf{r}_{k}-\mathbf{r}_{k}^{\mathrm{fcc}}\right)^{2}}\right]
$$

In this case, the unique fingerprint of a perfect fcc cubooctahedral coordination polyhedron consists of four $\overline{3}$ axes, hence the additional summation. Again, in constructing (2), the orientation of the four axes that most closely correspond to a local fcc-like environment has to be found by exhaustive search, this time in a three parameter (Euler angles) space. The set of all $\varepsilon_{i}^{\mathrm{fcc}}$ for all sites cumulated over the ensemble defines the norm distribution $\xi\left(\varepsilon^{\mathrm{fcc}}\right)$. Numerical constants in the definitions are the number of characteristic crystallographic elements ( 1 for hcp, 4 for fcc), and the number of elements distinct from unity in the subgroups associated with them (5 for both $\overline{6}$ and $\overline{3}$ ). These factors rescale the norms so that they can be compared fairly. In perfect hcp or fcc crystals, the corresponding norms $\varepsilon^{\text {hcp }}$ and $\varepsilon^{\text {fcc }}$ attain their minimum value of zero. Note that $\varepsilon^{\mathrm{hcp}}$ and $\varepsilon^{\mathrm{fcc}}$ detect both orientational and radial deviations from the perfect hcp or fcc coordination polyhedra. The distributions $\xi\left(\varepsilon^{\mathrm{hcp}}\right)$ and $\xi\left(\varepsilon^{\mathrm{fcc}}\right)$, especially 
their low- $\varepsilon$ part, are very sensitive to the fcc or hcp-like character of the surroundings of the site, and also exceedingly good indicators of MC relaxation [21]. The distributions $\xi\left(\varepsilon^{\text {hcp }}\right)$ and $\xi\left(\varepsilon^{\mathrm{fcc}}\right)$ show that, at densities far from the MRJ state, there exists a certain fraction of sites whose environment resembles a perfect hcp environment fairly well, and a smaller, completely disjoint fraction of sites [22] whose environment resembles a perfect fcc environment comparably well. The hcp dominance at intermediate values of the norms seems almost self evident, since it should be easier for a random environment to possess one single $\overline{6}$ axis than to possess four $\overline{3}$ axes simultaneously: the hcp coordination polyhedron has point group $\overline{6} m 2$ (order 12), while for more symmetrical fcc, point group is $m 3 m$ (order 48). However, the coordination polyhedra are clearly not random, and as the density approaches the MRJ state, fcc becomes dominant in the low-norm interval: $\xi\left(\varepsilon^{\mathrm{fcc}}\right)>\xi\left(\varepsilon^{\mathrm{hcp}}\right)$ for $\varepsilon^{\mathrm{fcc}}<0.2$; i.e., a small but increasing number of sites with almost perfect fcc-like environment appears, while sites with almost perfect hcp-like environment also exist but now they are fewer (than fcc-like) and their number grows more slowly. Thus, it can be stated that as the MRJ state is approached, a large fraction of sites possess fairly ordered ( $\varepsilon^{\mathrm{hcp}} \approx 0.5$ ) hcp-like environments. However, a small (but growing with $\varphi$ ) fraction of sites with almost perfect local order $\left(\varepsilon^{\text {hcp }}<0.2\right)$ can also be detected. The order of the environment around this small fraction of sites seems to possess somewhat predominant fcc character. For comparison purposes, we applied the same norms to single-sphere packings at the same density. Interestingly, it was found that: (a) the total number of sites with almost perfect order of any type ( $\varepsilon^{\mathrm{hcp}}$ or $\left.\varepsilon^{\mathrm{fcc}}<0.2\right)$ is higher for single hard sphere packings by roughly $30 \%$ and (b) most of this increase is due to the appearance of almost perfect hcp-like sites, while the number of almost perfect fcc-like sites increased only slightly.

Work supported in part by the EC through Contracts No. G5RD-CT-2002-00720 and No. NMP3-CT-2005016375, and by CICYT through Contract No. MAT200525569-E. Allocation of CPU time on the CeSViMa supercomputer "magerit" of UPM is gratefully acknowledged. We are especially indebted to one of the reviewers for pinpointing a major numerical error in Fig. 5.

*mlaso@industriales.upm.es

[1] S. Torquato, Random Heterogeneous Materials (SpringerVerlag, Berlin, 2002).

[2] S. Torquato, T. M. Truskett, and P. G. Debenedetti, Phys. Rev. Lett. 84, 2064 (2000).

[3] A. Donev, S. Torquato, and F. H. Stillinger, Phys. Rev. E 71, 011105 (2005).
[4] R. W. Chang and A. Yethiraj, Phys. Rev. Lett. 96, 107802 (2006).

[5] F. A. Escobedo and J.J. de Pablo, J. Chem. Phys. 102, 2636 (1995).

[6] K. Jagannathan, B. J. Sung, and A. Yethiraj, Phys. Rev. Lett. 97, 145503 (2006).

[7] J.P. Flory, Statistical Mechanics of Chain Molecules (Hanser Verlag, München, 1989).

[8] A. Donev, S. Torquato, and F. H. Stillinger, J. Comput. Phys. 202, 737 (2005).

[9] A. Donev, F. H. Stillinger, and S. Torquato, Phys. Rev. Lett. 95, 090604 (2005).

[10] T. W. Cochran and Y.C. Chiew, J. Chem. Phys. 124, 074901 (2006).

[11] M. Kotelyanskii and D. N. Theodorou, Simulation Methods for Polymers (Marcel Dekker, New York, 2004).

[12] M. Kröger, Comput. Phys. Commun. 118, 278 (1999); M. Kröger, M. Müller, and J. Nievergelt, CMESComput. Model 4, 559 (2003).

[13] P. V. K. Pant, D. N. Theodorou, Macromolecules 28, 7224 (1995); N.C. Karayiannis, V.G. Mavrantzas, and D. N. Theodorou, Phys. Rev. Lett. 88, 105503 (2002); J. I. Siepmann and D. Frenkel, Mol. Phys. 75, 59 (1992); J. J.de Pablo, M. Laso, and U.W. Suter, J. Chem. Phys. 96, 2395 (1992); M. P. Allen and D. J. Tildesley, Computer Simulation of Liquids (Clarendon, Oxford, 1989).

[14] N. C. Karayiannis and M. Laso, Macromolecules (to be published); preliminary evidence suggests the following conjecture: the density at the MRJ state is $\varphi_{\mathrm{hsc}}=\varphi_{\mathrm{ss}}$ for all chain lengths.

[15] Values for all observables obtained from different protocols agreed within 1 standard deviation in the mean. In addition, only fully decorrelated configurations were used: at the closest approach to the MRJ state, block length for statistical decorrelation of the radius of gyration was $5 \times$ $10^{6}$ steps, $5 \times 10^{8}$ steps for the distribution of torsional angles and $10^{9}$ steps for $\xi\left(\varepsilon^{\mathrm{hcp}}\right)$ and $\xi\left(\varepsilon^{\mathrm{fcc}}\right)$.

[16] J. G. Curro et al., J. Chem. Phys. 91, 1357 (1989); A. Yethiraj and C. K. Hall, J. Chem. Phys. 96, 797 (1992).

[17] J.P. Wittmer et al., Europhys. Lett. 77, 56003 (2007); P. Beckrich et al., Macromolecules 40, 3805 (2007).

[18] P. J. Steinhardt, D. R. Nelson, and M. Ronchetti, Phys. Rev. B 28, 784 (1983).

[19] L. V. Woodcock, Nature (London) 385, 141 (1997).

[20] W. Borchardt-Ott, Kristallographie (Springer-Verlag, Berlin, 2002).

[21] MC runs which seem to have reached equilibration when judged by all standard measures are often still far from equilibrated in terms of $\xi\left(\varepsilon^{\mathrm{hcp}}\right)$ and $\xi\left(\varepsilon^{\mathrm{fcc}}\right)$.

[22] Sites with low norms are either fcc-like or hcp-like; i.e., no site can simultaneously have low $\varepsilon^{\mathrm{fcc}}$ and $\varepsilon^{\text {hcp }}$ norms. The cce norms are strictly discriminating in this respect. At $\varphi=0.63$, fcc-like environments are more abundant by a factor of $\int_{0}^{0.23} \xi\left(\varepsilon^{\mathrm{fcc}}\right) d \varepsilon^{\mathrm{fcc}} / \int_{0}^{0.23} \xi\left(\varepsilon^{\mathrm{hcp}}\right) d \varepsilon^{\mathrm{hcp}}=$ $1.23 \pm 0.08$. 